\title{
On the origin of medium-period ionospheric waves and their possible modelling: a short review
}

\author{
Pietro Dominici $\left({ }^{1}\right)$, Ljiljana R. Cander $\left({ }^{2}\right)(*)$ and Bruno Zolesi $\left({ }^{3}\right)$ \\ (1) Dipartimento di Fisica, Università «La Sapienza», Roma, Italy \\ (2) Geomagnetic Institute, 11306 Grocka, Belgrade, Yugoslavia \\ (3) Dipartimento di Aeronomia, Istituto Nazionale di Geofisica, Roma, Italy
}

\begin{abstract}
This article introduces the concept of ionospheric waves with periods from about 15 min to about $4 \mathrm{~h}$ as one of the acoustic-gravity wave-induced phenomena. The existence of these medium-period ionospheric waves in the various ionospheric layers is supported by the results of a data analysis which has shown remarkable characteristics in occurrence and direction of the waves with a period not longer than about $2 \mathrm{~h}$. The explanation offered is based on the assumption that a unique phenomenon capable to launch acoustic-gravity waves related to such ionospheric waves is the sudden change in physical conditions of the atmosphere due to the passage of the solar terminator.
\end{abstract}

Key words ionosphere - ionospheric waves acoustic gravity waves

\section{Introduction}

The existence of movements in the upper atmosphere and hence in the ionosphere has been recognized since the early days of ionospheric research. Wave-like ionospheric structures clearly seen in any representation of space-time behaviour of ionospheric characteristics belong to the prominent atmospheric dynamics. A first description of these effects was given by Hines (1960), who stated that such structures are the ionospheric effects of atmo-

Mailing address: Dr. Bruno Zolesi, Istituto Nazionale di Geofisica, Via di Vigna Murata 605, 00143 Roma, Italy; e-mail: zolesi@marte.ingrm.it

(*) Now at: Rutherford Appleton Laboratory, Radio Communications Research Unit, Chilton, Didcot, Oxon OX11 0QX, U.K. spheric waves, namely ordinary compressional waves (acoustic waves) and a particular kind of transversal wave appearance (acoustic-gravity waves). Subsequent studies recognized that almost all large-amplitude and large-period (over $2 \mathrm{~h}$ ) southwards directed waves are launched by high-latitude phenomena of geomagnetic activity (e.g., Hunsucker, 1982). It is also showed that almost all small-amplitude and short-period (from a few minutes to few tenths of minutes) omnidirectional ionospheric waves are related to both acoustic and gravity waves launched by sudden atmospheric perturbations, like those due to thunderstorms, volcanic explosions, strong artificial explosions and earthquakes (e.g., Blanc, 1985). In the last few years, the trend is towards understanding the medium-period waves (between about $2 \mathrm{~h}$ and about $15 \mathrm{~min}$ ) (e.g., Dominici et al., 1988; Walker et al., 1988). Evidence has shown that these waves occur every day irrespective of geomagnetic activity with large amplitude during the day in wintertime and their direction ex- 
hibits a characteristic and regular variation during the day and year (Waldock and Jones, 1986). In spite of the large number of studies, however, at present there is no complete understanding of these waves either with respect to fundamental research or in connection with ionospheric modelling. Major questions remain concerning the detailed source mechanisms of acoustic-gravity waves and their modes of propagation.
This work extends previous studies of the ionosonde sounding results by Dominici et al. (1988) with the aim of introducing: i) the term Ionospheric Wave for the wave-like corrugations of the isoelectronic surfaces that in the past have been considered among Ionospheric Irregularities and then called Travelling Ionospheric Disturbances (TIDs); ii) the solar terminator as a possible source of generation of the medium-period ionospheric waves. For this
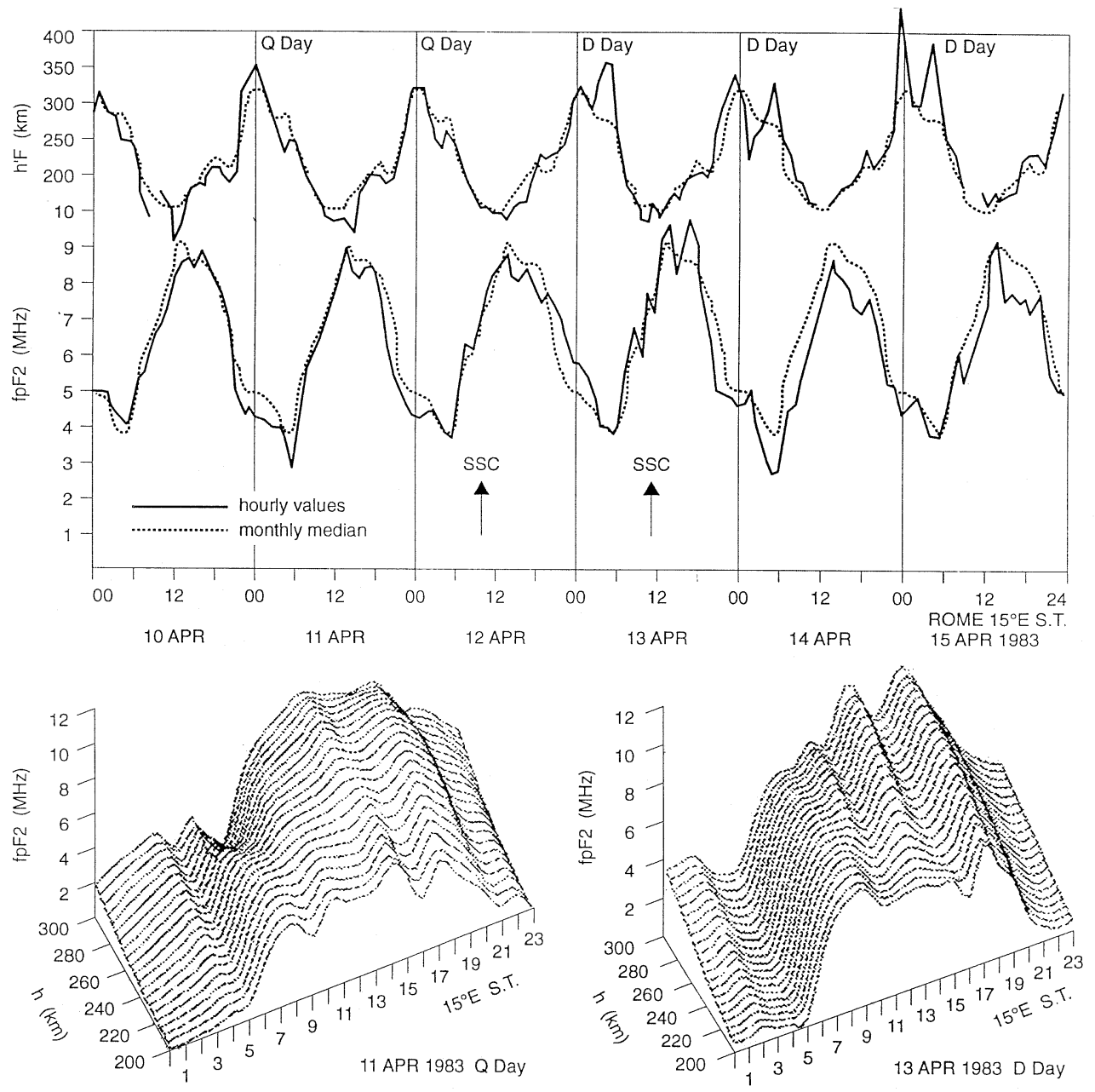

Fig. 1. Local appearance of ionospheric waves. Time behaviour of daily hourly values of $f_{o} F_{2}$ and $h^{\prime} F$ (top), and corresponding time-height pattern of the plasma frequencies $f_{p} F_{2}$ (bottom) at Rome ionospheric station during an equinoctial period including some geomagnetically quiet $(Q)$ and disturbed $(D)$ days. 
purpose, we examined critically some more data now available to show the close relation between such ionospheric waves and the solar terminator.

\section{Results}

Several studies have now demonstrated that the time behaviour of the ionosperic characteristics deduced from vertical-incidence ionograms (ordinary and extraordinary critical frequencies, virtual and real heights of the ionospheric layers) observed at any particular site always exhibit oscillations, the largest of which often seem to have some character of quasi-periodicity. Figure 1 shows the time behaviour of the ordinary critical frequency $f_{o} F_{2}$ and the virtual height $h^{\prime} F$ (top) and corresponding timeheight pattern of the plasma frequencies $f_{p} F_{2}$ (bottom) deduced by means of the Dudeney ionospheric model (Dudeney, 1978) from hourly ionograms taken at Rome ionospheric station during a period including geomagnetically quiet $(Q)$ and disturbed $(D)$ days. Indeed, two or three cycles of wide oscillations clearly appear superimposed on diurnal variation. But at this point it is necessary to recall that there are other important ionospheric irregularities which cannot be considered wave-like phenomena (Whitehead, 1970; Fejer and Kelley, 1980).

The fact that these oscillations at a particular ionospheric station can be considered or not as the local effect of the passage of a wave on a large spatial scale affecting the isoelectronic surfaces in the upperlying ionosphere depends on the existence of some coherence between the local ionospheric oscillations observed at the same time on other surrounding ionospheric stations. For instance, fig. 2 shows the

Fig. 2. Space-time variations of ionospheric waves. Time behaviour of daily hourly values of $f_{o} F_{2}$ and $D \%(-1+1)$ at three European ionospheric stations during a winter period including geomagnetically quiet and disturbed days. Corresponding values of the $A E$ index are shown on the top.

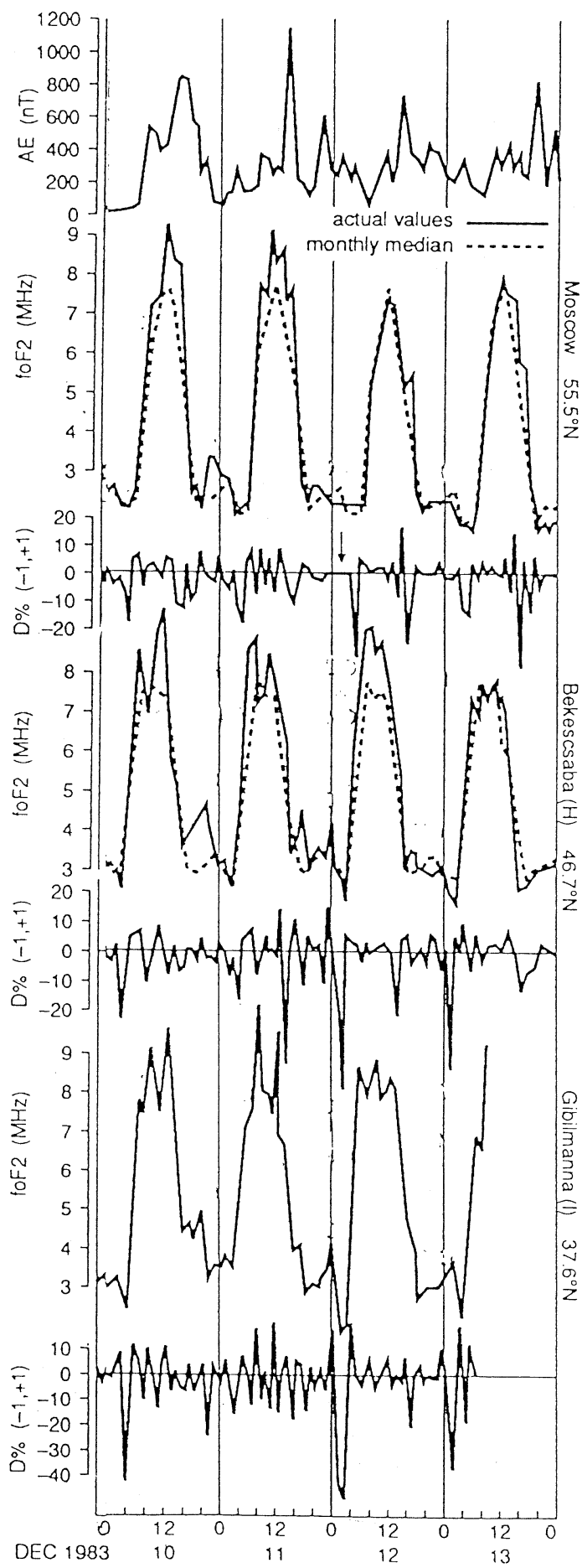



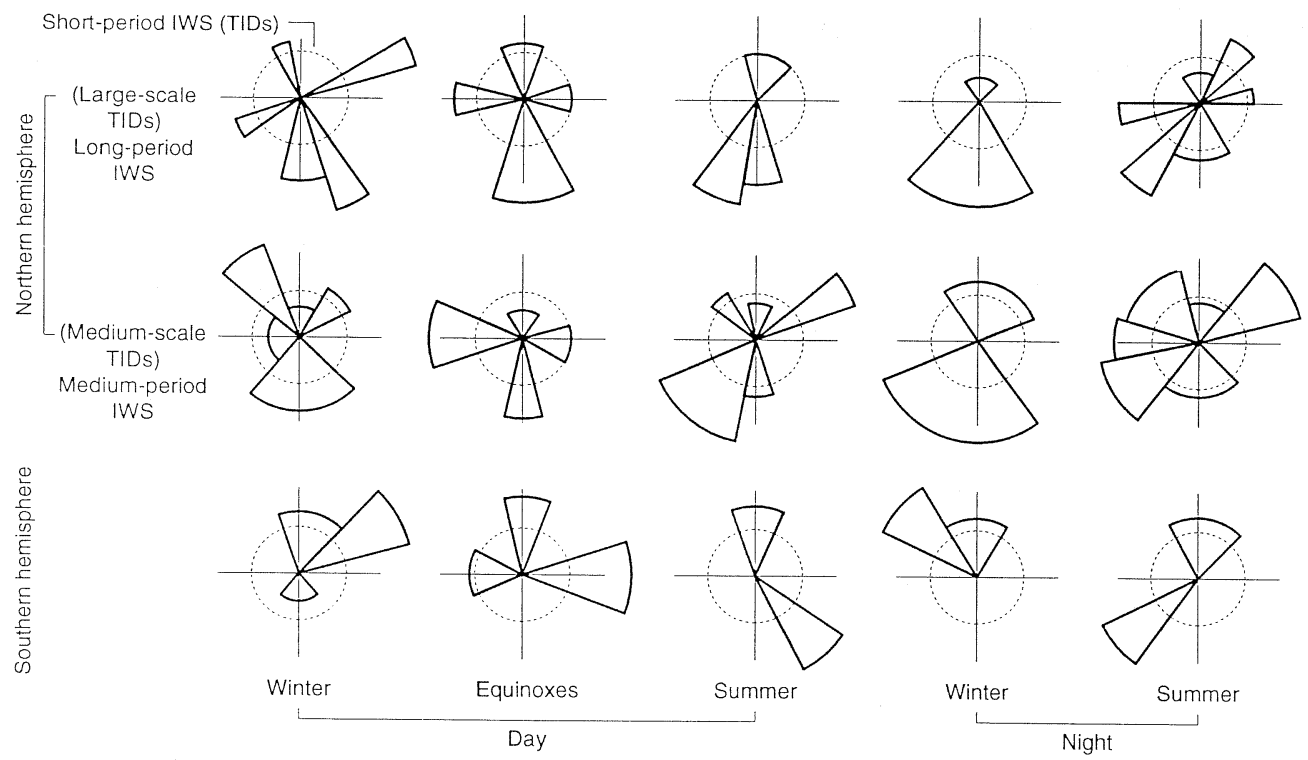

Fig. 3. Distribution of directions of long- and medium-period ionospheric waves as averaged from the available data. The dotted circles represent the omnidirectional short-period ionospheric waves.

time behaviour of hourly values for $f_{o} F_{2}$ and of the corresponding oscillatory component as a relative deviation from the 3 -value symmetrical running mean $D \%(-1+1)$ at three European ionospheric stations during a winter period including geomagnetically quiet and disturbed days. The geomagnetic activity is indicated by the auroral electrojet index $A E$. It is clear that the medium-period ionospheric waves are always present at daytime even in periods of low geomagnetic activity (9/10 December 1983), and seem to be ambler as the latitude decreases, while night-time oscillations are related to the beginning and the development of geomagnetic activity, and merge into daytime oscillations.

In spite of the frequency with which acoustic-gravity waves are invoked to explain wavelike variation in data, their morphology is not well documented, and apparently conflicting results exist in the experimental literature. This is mostly due to the difficulty of measuring a sufficient number of wave parameters to make a meaningful assessment of their significance and to permit an examination of their interrelationships. Using the most homogeneous data available, a general average pattern of ionospheric waves is given in fig. 3. In this figure the similarly averaged daytime/night-time direction distribution is shown. Some conclusions can be added to those directly resulting from the figure as follows: i) the constantly present northward or southward components regard very-long period ionospheric waves; ii) the circles in the polar diagrams regard the always present random-directed small-amplitude waves of the background noise, and iii) the remaining part which exhibits the amplest seasonal and diurnal variation regards mediumperiod ionospheric waves. The prevalent direction of these waves changes during the daytime from $\mathrm{NE}$ in winter to $\mathrm{SE}$ in summer in the southern hemisphere, being eastward around the equinoxes. The situation seems much more complicated, especially at night-time in northern hemisphere, but it is simply due to the larger amount of data we have had from northern hemisphere stations than from southern 
hemisphere ones. An orthogonal change seems to occur, in any particular site, between daytime and night-time directions.

\section{Discussion and conclusions}

It is well known that the solar terminator passes twice per day at each point of the Earth's surface, at sunrise (morning terminator) and sunset (evening terminator). The nomo- graphs of fig. 4 give the local time of sunrise and sunset at middle latitudes for the 15th day of each month at 0,100 and $300 \mathrm{~km}$ of altitude. This figure also gives an idea of the form of the solar terminator's surface near the Earth's surface.

The atmospheric conditions differ in day and night hemispheres, apart from the solar illumination, because of the different values of physical parameters, such as the temperature and the number density of the various neutral and charged particles in the ionosphere. There-
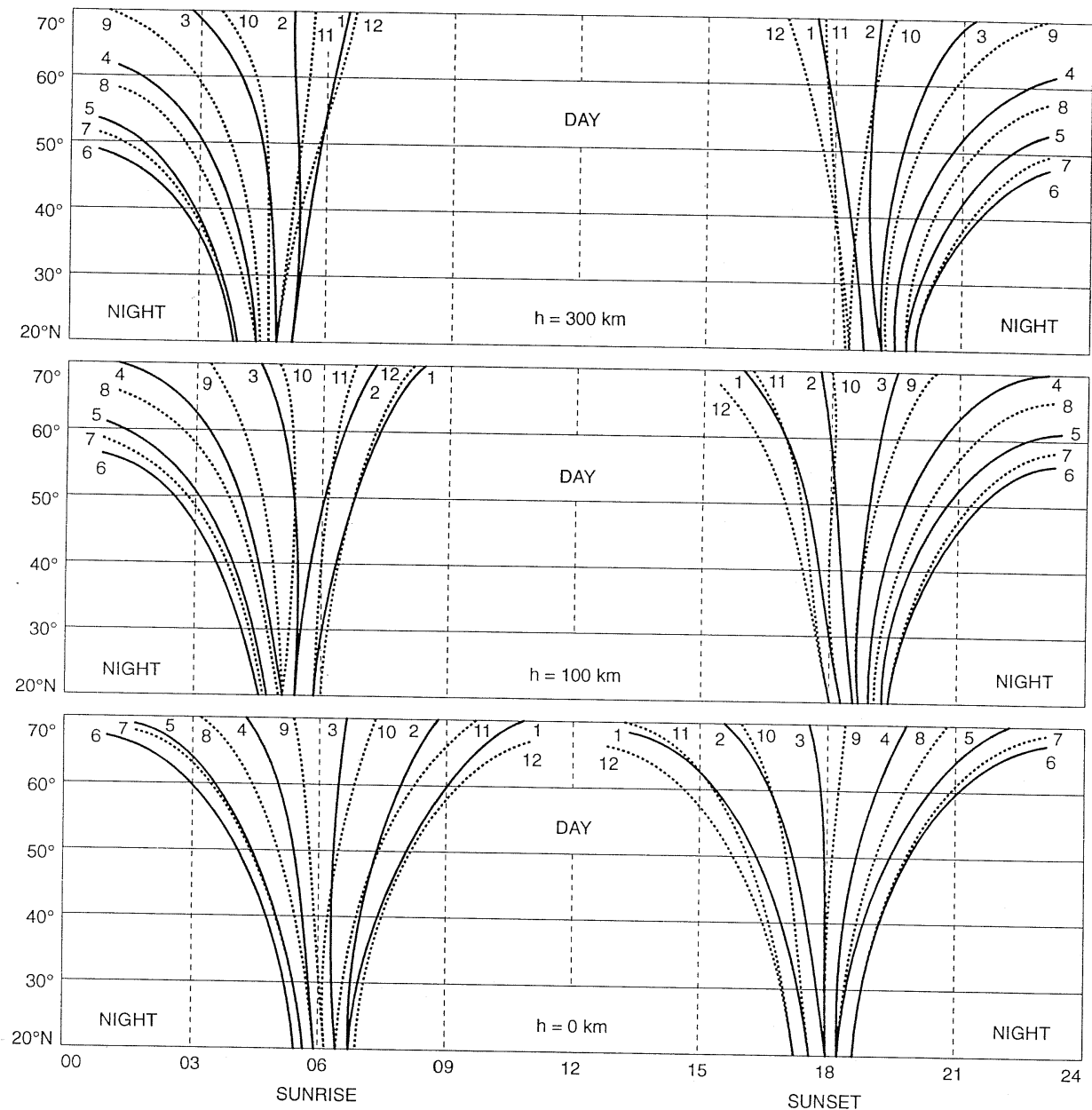

Fig. 4. Local time of sunrise and sunset at mid-latitude on 15 th day of each month ( 1 = January; 2 = February, etc.) at 0,100 and $300 \mathrm{~km}$ of altitude. 
fore, from a physical point of view the morning terminator is a three-dimensional surface where at each height a transition occurs from night conditions (absence of direct solar photons, low temperature, etc.) to day conditions (presence of direct flux of solar photons, higher temperature, etc.) and analogously for the evening terminator. The solar terminator can be consequently considered the spatial region where a sufficiently high gradient of one or more of the above physical parameters exists. So, the problem of what the solar terminator is becomes the capital problem of how large a gradient must be considered in defining it. At present, there is no general agreement on that, although some tentative suggestions have been made (e.g., Somsikov, 1991).

Once the significant parameter has been chosen, its gradient field or if one prefers its space distribution defines the «ionospheric width» of the solar terminator. This width has an important role for the efficiency of the solar terminator in launching atmospheric waves.
This efficiency is larger the smaller the width, reaching a maximum for an infinitesimal width (infinite gradient), to which corresponds the ideal switch-on function for the source function based on the selected parameter. From a purely ionospheric point of view, a rough, but at first sufficiently significant, estimate of the width of the physical solar terminator can be given by the time derivative at dusk and dawn of $f_{o} F_{2}$, that can be seen as a local measure of the zonal gradient of $f_{o} F_{2}(1 \mathrm{MHz} / \mathrm{h}$ corresponds to $0.6 \mathrm{~s} \phi \mathrm{kHz} / \mathrm{km}$, being $\phi$ the latitude). From fig. 5a, which is sufficiently indicative for all mid-latitude situations, we can observe that in winter periods this «ionospheric width» of the morning terminator is always larger than that of the evening terminator (by a factor of about 1.5) and that in summer the two widths are much smaller than the corresponding ones in winter (by a factor of about 6). Other indications are present in fig. 5b, depicting the more favourable situation existing in winter and at sunrise to the atmospheric grav-

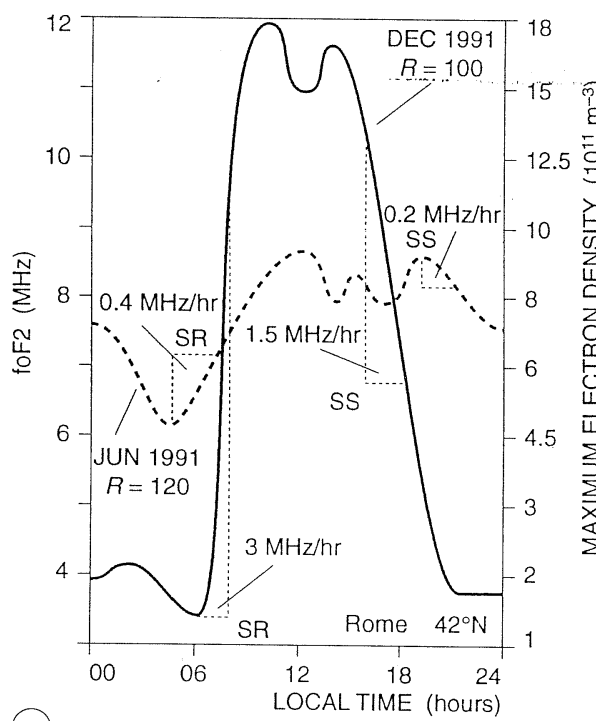

(a)

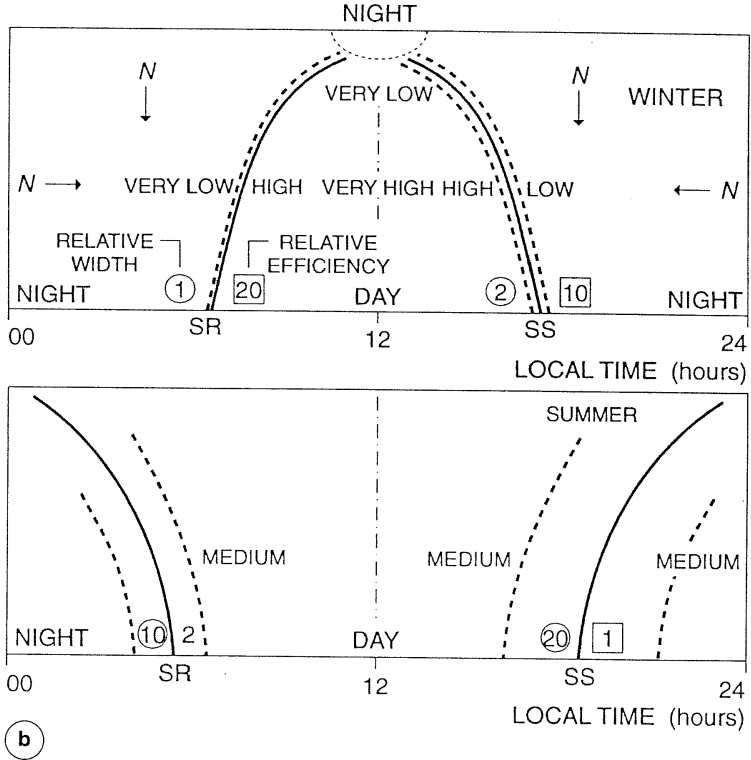

(b)

Fig. 5a,b. A scheme of the physical solar terminator with definition of «ionospheric width» (a) and some related physical conditions in winter and summer (b). The arrows indicated the directions of increasing electron density $N$. 
ity waves launched by the solar terminator. It is also important to note that there is another anisotropy in the ionospheric waves launched by the solar terminator, namely the different efficiency of the day and night hemisphere in propagating ionospheric waves. Although we still do not have at our disposal a satisfactory magnetohydrodynamical (MHD) theory for the propagation of the ionospheric waves, a reasonable conjecture is in favour of a better efficiency of the day atmosphere because of its minor total MHD viscosity. All that can explain, at least in part, the observed larger evidence of medium-scale ionospheric waves after sunrise in winter.

Therefore, atmospheric gravity waves and then ionospheric waves launched by the more or less thin region we have called physical solar terminator, but in the present considerations

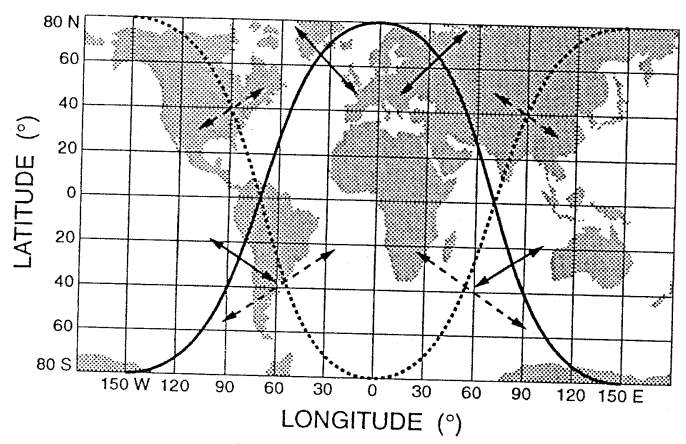

Fig. 6. A scheme of the worldwide position of the solar terminator on the Earth's surface in December (solid line) and June (dotted line) at 1200 GMT. The direction and length of the arrows roughly indicate respectively the direction (neglecting any wind influence) and efficiency in acoustic-gravity wave launching.

Table I. Data on directions of medium-period ionospheric waves.

\begin{tabular}{|c|c|c|c|c|c|c|}
\hline \multirow[t]{2}{*}{ Authors } & \multicolumn{3}{|c|}{ Daytime (h) } & \multicolumn{3}{|c|}{ Night-time } \\
\hline & Winter & Equinox & Summer & Winter & Equinox & Summer \\
\hline & \multicolumn{6}{|c|}{ Northern hemisphere } \\
\hline Bramley (1953) & $\begin{array}{c}\mathrm{SE}(<14 \mathrm{~h}) \\
\mathrm{SSW}(>14 \mathrm{~h})\end{array}$ & & & & & \\
\hline Crowley and McCrea (1988) & $\mathrm{SW}(>12 \mathrm{~h})$ & & & & & \\
\hline Davies and Jones (1971) & $\mathrm{SSW}^{*}$ & $\mathrm{~W}^{*}$ & $\mathrm{NNW}^{*}$ & & & \\
\hline Drobzhev et al. (1988) & SW & & & NW & $\mathrm{NE}$ & \\
\hline Ivanov et al. (1987) & $\begin{array}{l}\mathrm{SE}(<12 \mathrm{~h}) \\
\mathrm{SW}(>12 \mathrm{~h})\end{array}$ & $\mathrm{E}$ & $\mathrm{NE}$ & $\mathrm{NW}^{* *}$ & $\mathrm{~S}^{* *}$ & $\mathrm{SE}^{* *}$ \\
\hline Jacobson and Ericson (1992) & \multicolumn{3}{|c|}{ SE, S, SW } & & SW, E & \multirow{5}{*}{ NE-NW } \\
\hline Liyadze and Sharadze (1987) & SE & $\mathrm{E}$ & $\mathrm{NE}$ & & & \\
\hline Sharadze et al. (1987) & & & SE-SW & & & \\
\hline Thomas (1959) & SE & & E & & & \\
\hline Waldock and Jones (1986) & $\begin{array}{l}\mathrm{SE}(<12 \mathrm{~h}) \\
\mathrm{S}(\approx 12 \mathrm{~h}) \\
\mathrm{SW}(>12 \mathrm{~h})\end{array}$ & $\mathrm{E}$ & $\mathrm{NE}$ & & & \\
\hline
\end{tabular}

Munro (1958)

Heisler (1963)

Titheridge (1969)
$\mathrm{NE}$

$\mathrm{NE}$

\section{Southern hemisphere}

$\mathrm{E}$

SE

SE
SW

MW

* Near sunset; ** near sunrise. 
we shall approximate this source region by its central part, i.e., the surface of the geometrical solar terminator. Even referring to some of the above conclusions on the solar eclipse effects, one can say that the observed ionospheric waves derived from atmospheric gravity waves launched by each element of the solar terminator, acting as a point-like source, and from the successive interference among these elementary waves, so that the form of the wavefront repeats that of the solar terminator. In fig. 6 the solar terminator profile at ground level in December and June is reported on a worldwide scale. Some hypothetical wave directions, lying along the horizontal trace of the perpendicular to the solar terminator surface, are also indicated by arrows, their length being roughly indicative of the relative efficiency in related wave launching. As we recalled above, the wave launching is more efficient towards the day hemisphere than towards the night hemisphere. So, in day time hours before the solar noon the observed ionospheric waves come from the morning terminator, western, and the same is for the early hours in the afternoon, but near sunset perhaps waves from the evening terminator, eastern, can be observed. In winter daytime, when the two terminators are relatively close to each other, equatorward waves around noon and zonal standing waves near sunset cannot be excluded, especially at moderately high latitude.

These findings can explain some details of the apparent scatter in directions we previously evidenced in fig. 3. In this regard, because the question of the distribution of direction of these waves seems to be of crucial importance, we have revised all the unambiguous experimental results and classified them in relation to the season and hour of the day, as table I shows. So, the scattered direciton pattern in the part of fig. 3 concerning the directions of medium-period ionospheric waves are now resolved in a pattern which is highly coherent with the assumption that the wave direction is orthogonal to the nearest and most efficient solar terminator. This experimental evidence can be considered a good starting point in better understanding the role of the solar terminator in launching the ionospheric waves.

\section{REFERENCES}

BlanC, E. (1985): Observations in the upper atmosphere of infrasonic waves from natural or artificial sources: a summary, Ann. Geophys., 3, 673-688.

BRAMLEY, E.N. (1953): Direction-finding studies of large scale ionospheric irregularities, Proc. R. Soc. London, 229, 39.

Crowley, L.N. and I.W. MCCREA (1988): A synoptic study of TIDs observed in the U.K. during the first WAGS campaign, Radio Sci., 23, 905-917.

DAvieS, K. and J.E. Jones (1971): Three-dimensional observations of TIDs, J. Atmos. Terr. Phys., 33, 39-46.

DUDENEY, J.R. (1978): An improved model of the variation of electron concentration with height in the ionosphere, J. Atmos. Terr. Phys., 40, 195-203.

Dominici, P., LJ. R. CANDER and B. Zolesi (1988): Preliminary results concerning AGWs deduced from $f_{o} F_{2}$ large-scale oscillations, Physica Scripta, 37, 516-522.

DrobZHEV, V.I., Yu. G. LITVINOV and A.F. YAKOVETS (1988): Phase velocities of medium-scale wave disturbances of the ionosphere, Geomagn. Aeron., 28, 258-260.

FeJER, B.G. and M.C. KELLEY (1980): Ionospheric irregularities, Rev. Geophys. Space Phys., 18, 401-454.

HEISLER, L.H. (1963): Observation of movement of perturbations in the F-region, J. Atmos. Terr. Phys., 25, 71-86.

HiNES, C.O. (1960): Internal atmospheric gravity waves at ionospheric heights, Can. J. Phys., 53, 1441.

HUNSUCKER, R.D. (1982): Atmospheric gravity waves generated in the high-latitude ionosphere: a review, Rev. Geophys. Space Phys., 20, 293-315.

IVANOV, V.P., V.L. KARVETSKIY and N.A. KoREN'KOVA (1987): Seasonal-daily variations in parameters for medium-scale moving ionospheric disturbances, Geomagn. Aeron., 27, 446-448.

JACOBSON, A.R. and W.C. ERICSON (1992): Wavenumberresolved observations of ionospheric waves using the very large array radiotelescope, Planet. Space Sci., 40, 447-455.

LIYADZE, A.L. and Z.B. SHARADZE (1987): Simultaneous investigations of small and large inhomogeneities $F$ region, Geomagn. Aeron., 27, 646-649.

MunRo, G.H. (1958): Travelling disturbances in the $F$ region, Aust. J. Phys., 11, 91.

ShaRADZE, Z.S., Z.H. G. Gogiashvili, N.V. Mosashvili and O.P. CHOKHELI (1987): Interrelation of wave disturbances in the $E$ and $F$ regions of the mid-latitude ionosphere, Geomagn. Aeron., 27, 577-580.

Somsikov, V.M. (1991): Waves in the atmosphere due to the solar terminator (Review), Geomagn. Aeron., 31, 1-8.

Thomas, L. (1959): Some measurements of horizontal movements in region $F_{2}$ using widely spaced observing stations, J. Atmos. Terr. Phys., 14, 123.

TITHERIDGE, J.E. (1969): A regular disturbance in the topside ionosphere, J. Geophys. Res., 74, 1195-1204.

WALDOCK, J.A. and T.B. JONES (1986): HF Doppler observations of medium-scale TIDs at mid-latitudes, J. Atmos. Terr. Phys., 48, 245-260.

WALKER, G.O., W.Y. WONG, J.H.K. MA, T. KIKUCHI, K NoZAKI, Y.N. HuANG and V. BADILlo (1988): Propagating ionospheric waves observed throughout East Asia during the Wags October 1985 campaign, Radio Sci., 23, 867-878.

WhITEHEAD, J.D. (1970): Production and prediction of sporadic E, Rev. Geophys. Space Phys., 8, 65-144. 